MODELING, IDENTIFICATION AND CONTROL, 1983, VOL. 4, NO. 4, 223-235

doi:10.4173/mic.1983.4.3

\title{
Robustness analysis of a class of optimal control systems
}

\author{
OLE A. SOLHEIM $\dagger$ \\ Keywords: Optimal control systems, robustness analysis. \\ The paper deals with a class of optimal control systems, where the controller, \\ in addition to minimizing a quadratic criterion, also shall give the closed-loop \\ system prescribed eigenvalues. Three methods for analysing the robustness of \\ such systems under parameter perturbations are discussed: Eigenvalue sensitivity, \\ singular values and the block Gerschgorin theorem. Numerical examples are \\ presented to illustrate the different methods.
}

\section{Introduction}

A fundamental requirement of any practical feedback control system is its stability robustness, that is, the ability to maintain stability in the face of modeling errors. The design of the controller has to be based on a mathematical model of the real process. There may, however, be errors both in parameters and structure of the model. How is it now possible to bring such model uncertainties into the design process? Let us concentrate on one important issue: stability. In order to allow for uncertainties in the model and also for variations in the process, the design must have a safety margin as to stability.

When designing multivariable systems in the frequency domain, the robustness specifications may be included directly in the design process, for example by the use of phase margins, singular value plots, etc. (Doyle and Stein 1981, Lee et al. 1982, Lehtomaki et al. 1981 a, b, MacFarlane 1981).

When designing multivariable systems in the time domain, one method to include stability robustness in the design process, is to reduce eigenvalue sensitivity to parameter variations. In Shah et al. (1977) it is shown how the closed-loop eigenvalues can be made invariant to unknown perturbations in system parameters. One limitation of this method is that only some of the eigenvalues can be made insensitive to variations in some of the parameters. The sensitivity to the rest of the parameters may be rather large (see Example 1 in §3). In Gourischankar and Ramar (1976) one considers eigenvalue placement with minimum sensitivity to parameter variations. The same limitations as mentioned above exist here also (see Example 1 in $\S 3$ ). Further investigations of reducing eigenvalue sensitivities are described in Heger and Frank (1982), Howze and Carin (1979) and Sambandan and Chandrasekharan (1981).

The linear quadratic regulator (LQR) is known to have good robustness properties (Lehtomaki et al. 1981 a). For example, the eigenvalue sensitivities of systems with this type of controller are rather low, as is demonstrated below in Example 1. It is also possible to design the LQR so that desired closed-loop eigenvalues can be

Received 15 August 1983.

$\dagger$ Division of Engineering Cybernetics, The Norwegian Institute of Technology, N-7034, Trondheim-NTH, Norway. 
obtained (Graupe 1972, Solheim 1972, 1980, Taylor and Tuteur 1966) or that they can be located in desired regions in the complex plane (Andersen and Moore 1971). Thus we may avoid having eigenvalues too close to the imaginary axis.

Several methods cxist for the analysis of the robustness of feedback systems. In the present paper we shall deal with three different methods:

(1) Eigenvalue sensitivity $(\S 3)$

(2) Singular values $(\S 4)$

(3) The block Gerschgorin theorem $(\S 5)$.

But before going into a detailed discussion of these methods, let us first consider the design problem more closely.

\section{Problem statement}

Consider the time-invariant linear process

$$
\left.\begin{array}{l}
\dot{x}=A x+B u \\
y=D x
\end{array}\right\}
$$

where $x, u$ and $y$ are $n$-dimensional state, $r$-dimensional control and $m$-dimensional measurement vectors, respectively.

We use the usual quadratic performance index

$$
J=\frac{1}{2} \int_{0}^{\infty}\left[x^{\mathrm{T}} Q x+u^{T} P u\right] d t
$$

where $Q$ is a symmetric non-negative definite matrix and $P$ is a symmetric positive definite matrix.

Let us in the first place assume that all state variables are directly accessible. We therefore set $y=x$, that is, the measurement matrix $D=I$.

In addition to requiring the minimization of the performance index, we also require that the closed-loop system

based on the control law

$$
\dot{x}=(A+B G D) x=F x
$$

$$
u=G y=G D x
$$

attains prescribed eigenvalues.

In order to achieve this, the weighting matrix $Q$, for the state, cannot be freely chosen, but must be determined so that the prescribed eigenvalues are obtained (Solheim 1972, 1980).

Let us consider the following parameter perturbations.

(i) Change $d A$ in the process matrix $A$ due to process parameter variations.

(ii) Change $d B$ in the control matrix $B$ due to variations in process and actuator parameters.

(iii) Change $d D$ in the measurement matrix $D$ due to sensor parameter variations.

The total change $d F$ in the closed-loop matrix $F$ becomes (with $D=I$ )

$$
d F=d A+d B \cdot G+B G \cdot d D
$$

In the present paper we shall consider additive perturbations only. 
The purpose of the robustness analysis is now to indicate the size of the perturbations that can be tolerated without endangering the stability of the system. Or, in other words, how a given change in the parameters influences the eigenvalues of the system.

Before we go into a detailed discussion of this problem let us also consider the case where all states are not directly measurable. In this case a state estimator has to be included in the controller.

Let $\hat{x}$ represent the estimated state. With the control law

$$
u=G \hat{x}
$$

we get the total closed-loop system

$$
\left[\begin{array}{c}
\dot{x} \\
\hat{x}
\end{array}\right]=\left[\begin{array}{c:c}
A & B G \\
\hdashline K D & A_{m}+B_{m} G-K D_{m}
\end{array}\right]\left[\begin{array}{l}
x \\
\hat{x}
\end{array}\right]=\hat{F}\left[\begin{array}{l}
x \\
\hat{x}
\end{array}\right]
$$

where $K$ is the estimator gain, and subscript $m$ indicates matrices used in the estimator.

Introducing the estimation error $\Delta x=x-\hat{x}$ the system (7) may be expressed as

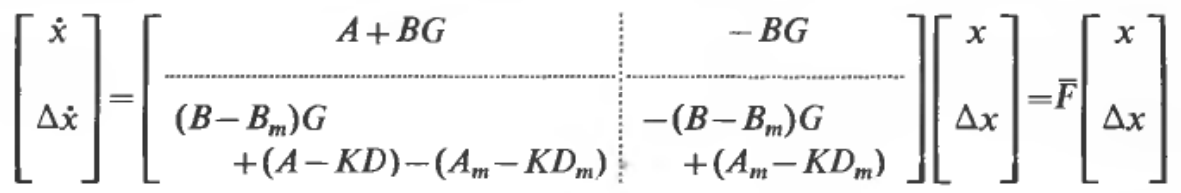

When the model used in the estimator exactly matches the process, (8) simplifies to

$$
\left[\begin{array}{c}
\dot{x} \\
\Delta \dot{x}
\end{array}\right]=\left[\begin{array}{c:c}
A+B G & -B G \\
\hdashline 0 & A-K D
\end{array}\right]\left[\begin{array}{c}
x \\
\Delta x
\end{array}\right]=\bar{F}\left[\begin{array}{c}
x \\
\Delta x
\end{array}\right]
$$

Assuming changes in $A, B$ and $D$ of the process (no changes in the model used in the estimator), the changes in $\hat{F}$ and $\bar{F}$ become

$$
\begin{aligned}
& d \hat{F}=\left[\begin{array}{c:c}
d A & d B G \\
\hdashline K d D & 0
\end{array}\right] \\
& d \bar{F}=\left[\begin{array}{c:c}
d A+d B G & -d B G \\
\hdashline d A+d B G-K d D & -d B G
\end{array}\right]
\end{aligned}
$$

From $(8 a)$ we note that the eigenvalues of the total system $\bar{F}$ consist of the eigenvalues of the closed-loop system $(A+B G)$, plus the eigenvalues of the estimator $(A-$ $K D)$. These two parts may therefore be designed separately. In the same way that we may design an optimal feedback system with prescribed eigenvalues, we may also design an optimal estimator with prescribed eigenvalues (Solheim 1972). This means that we have full control of the stability of the total system, that is, when the real and the nominal parameters agree.

For the subsequent investigations the analysis will be greatly simplified if all eigenvalues are real. Using the above mentioned design method (Solheim 1972) this is easily achieved. 


\section{Eigenvalue sensitivity}

A small change $d A$ in a matrix $A$ produces a small first order change in the eigenvalue $\lambda_{i}$ (see for example (Faddeev and Faddeeva 1963))

$$
d \lambda_{i}=n_{i} d A m_{i}
$$

where $n_{i}$ is an eigenrow and $m_{l}$ is an eigenvector of $A$ corresponding to $\lambda_{l}$.

The sensitivity coefficients may be obtained from (11) by partial differentiation

$$
\frac{\partial \lambda_{l}}{\partial a_{k l}}=\left(n_{i}\right)_{k}\left(m_{i}\right)_{l}
$$

where $a_{k l}$ is an element of $A$.

Consider now the closed-loop system (3) with the perturbations (5). The change in the closed-loop eigenvalue $\lambda_{i}$ becomes

$$
d \lambda_{l}=n_{l}(d A+d B G+B G \cdot d A) m_{i}
$$

where $n_{i}$ and $m_{i}$ are, respectively, an eigenrow and an eigenvector of $F$.

We mentioned above that no method exists for the inclusion of eigenvalue sensitivities directly in the LQR design. Such controllers have, however, rather good properties in this respect as is demonstrated below in Example 1. We compare here with two other methods where reduction of the eigenvalue sensitivity is part of the design process.

\section{Example 1}

Given the system

$$
A=\left[\begin{array}{lll}
0 & 1 & 0 \\
0 & 1 & 1 \\
0 & 0 & 1
\end{array}\right], \quad B=\left[\begin{array}{ll}
1 & 0 \\
0 & 1 \\
1 & 0
\end{array}\right], \quad D=\left[\begin{array}{lll}
1 & 0 & 0 \\
0 & 1 & 0 \\
0 & 0 & 1
\end{array}\right]
$$

The eigenvalues of the closed-loop system are specified as: $-1,-2,-3$. Using an LQR this is achieved with the weighting matrices

$$
P=\left[\begin{array}{ll}
1 & 0 \\
0 & 1
\end{array}\right], \quad Q=\left[\begin{array}{rrr}
4.9 & 5.7 & -9.4 \\
5.7 & 7.6 & -10.3 \\
-9.4 & -10.3 & 18.4
\end{array}\right]
$$

The sensitivity coefficients of the dominant eigenvalue $\lambda_{1}=-1$ are shown in Table 1 .

To evaluate the robustness properties of the optimal controller, we propose the following method. With a given optimal controller we compute the sensitivity coefficients of the dominant eigenvalue of the closed-loop matrix $F$. The sign of the coefficients indicates in which direction the eigenvalue will move for a change in the corresponding parameter. Using estimated maximum magnitudes of the parameter variations, and sign chosen so that all variations will move the dominant eigenvalue towards the imaginary axis, a set of 'worst case' parameters is obtained. We then compute the eigenvalues of the perturbed system. If the stability is not good enough we may specify new nominal eigenvalues and repeat the analysis until we arrive at a satisfactory system. The procedure is illustrated below in Example 2. 


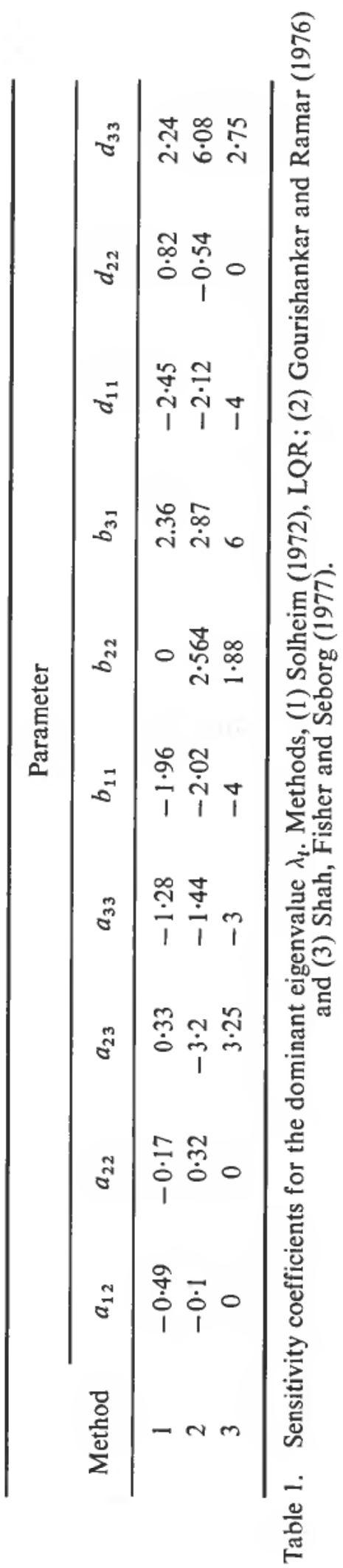


Example 2

Given the system

$$
A=\left[\begin{array}{rr}
-2 & 0 \\
1 & -1
\end{array}\right], \quad B=\left[\begin{array}{ll}
1 & 0 \\
0 & 1
\end{array}\right], \quad P=\left[\begin{array}{ll}
1 & 0 \\
0 & 5
\end{array}\right], \quad D=\left[\begin{array}{ll}
1 & 0 \\
0 & 1
\end{array}\right]
$$

The eigenvalues of the closed-loop system are specified as $-5,-8$. Using an LQR this is obtained with

$$
Q=\left[\begin{array}{cc}
62 \cdot 2 & 15 \cdot 6 \\
15 \cdot 6 & 109 \cdot 0
\end{array}\right], \quad G=\left[\begin{array}{cc}
-6 \cdot 37 & -2 \cdot 6 \\
-0.52 & -3 \cdot 63
\end{array}\right]
$$

The sensitivity coefficients of the dominant eigenvalue at -5 are shown in Table 2 .

Parameter

$\begin{array}{llllllll}a_{11} & a_{21} & a_{22} & b_{11} & b_{22} & d_{11} & d_{22} & d_{12}\end{array}$

Without

estimator

$\begin{array}{llllllll}\text { Example } 2 & -0.12 & -0.87 & 1.12 & 0.37 & -3.63 & 1.24 & -4.5\end{array}$

With

estimator

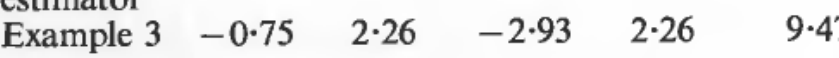

Table 2. Sensitivity coefficients for the dominant eigenvalue $\lambda_{1}=-5$.

Assuming a $20 \%$ variation in all parameters in $A, B$ and $D$, and choosing the sign of the variations so that a 'worst case' is obtained, we get

$$
\Delta A=\left[\begin{array}{cc}
-0.4 & 0 \\
-0.2 & 0.2
\end{array}\right], \quad \Delta B=\left[\begin{array}{cc}
0.2 & 0 \\
0 & -0.2
\end{array}\right], \quad \Delta D=\left[\begin{array}{cc}
0.2 & 0 \\
0 & -0.2
\end{array}\right]
$$

The eigenvalues of the closed-loop system $[(A+\Delta A)+(B+\Delta B) G(D+\Delta D)]$ become $-3 \cdot 2,-11 \cdot 48$.

If the eigenvalue at $-3 \cdot 2$ is not acceptable, we may try another design with, for example, nominal eigenvalues at $-6,-10$. With 'worst case' perturbations we get for this new system the eigenvalues -3.92 and -13.94 .

Next we consider a system with a state estimator, eqns. (7) or (8). Let us denote the change in an eigenvalue of the feedback part of the system by $d \lambda_{i}{ }^{F}$. We may split this change into two parts so that the influence of the estimator is more clearly revealed (Solheim and Sælid 1971). The model matrix (and its inverse) of the system ( $8 a$ ) can be written

$$
\bar{M}=\left[\begin{array}{c:c}
M^{F} & M^{c} \\
\hdashline 0 & M^{E}
\end{array}\right], \quad \bar{M}^{-1}=\left[\begin{array}{c:c}
N^{F} & N^{c} \\
\hdashline 0 & N^{E}
\end{array}\right]
$$


where $M^{F}$ is the modal matrix of the feedback system $(A+B G)$ and $M^{E}$ of the estimator $(A-K D)$. From (Solheim and Sælid 1974) we get

$$
d \lambda_{i}{ }^{F}=n_{i}{ }^{F}[d A+d B G] m_{i}{ }^{F}+n_{i}{ }^{C}[d A+d B G-K d D] m_{i}{ }^{F}
$$

where $n_{i}{ }^{F}$ and $m_{i}{ }^{F}$ are eigenrows and eigenvectors of $(A+B G)$, and $n_{i}{ }^{C}$ is a row of the matrix $N^{C}$ in (13).

The first term on the right-hand side of (14) is the eigenvalue change we get without estimator, and the second term represents the additional change due to the estimator.

We may also obtain an expression for the eigenvalue change in the estimator part of the total system, but since the dominant eigenvalues are supposed to be in the feedback part, we omit that here.

\section{Example 3}

Given the system

$$
A=\left[\begin{array}{rr}
-2 & 0 \\
1 & -1
\end{array}\right], \quad B=\left[\begin{array}{ll}
1 & 0 \\
0 & 1
\end{array}\right], \quad D=\left[\begin{array}{ll}
0 & 1
\end{array}\right]
$$

with

$$
G=\left[\begin{array}{cc}
-6.37 & -2 \cdot 6 \\
-0.52 & -3.63
\end{array}\right] \text { and } K=\left[\begin{array}{l}
80 \\
19
\end{array}\right]
$$

we get the specified eigenvalues

$$
\lambda_{1}{ }^{F}=-5, \quad \lambda_{2}{ }^{F}=-8, \quad \lambda_{1}{ }^{E}=-10, \quad \lambda_{2}{ }^{E}=-12
$$

The eigenvalue sensitivities are computed and, for the sake of comparison, shown in Table 2. We note that the sensitivities for this case are somewhat larger than for the case without estimator.

Assuming a $10 \%$ change in all parameters the 'worst case' changes become

$$
d A=\left[\begin{array}{cc}
-0 \cdot 2 & 0 \\
0 \cdot 1 & -0 \cdot 1
\end{array}\right], \quad d B=\left[\begin{array}{cc}
0 \cdot 1 & 0 \\
0 & 0 \cdot 1
\end{array}\right], \quad d D=\left[\begin{array}{ll}
0 & 0 \cdot 1
\end{array}\right]
$$

The eigenvalues of the total system with the perturbed parameters are $-4 \cdot 2,-8 \cdot 8 \pm$ $j 5 \cdot 8,-13 \cdot 5$.

We have shown in the examples above how we may use sensitivity coefficients to evaluate system robustness. These coefficients can also be used in selecting the critical parameters in the process, so that we may try to model these parameters more accurately and also try to avoid large variations in them.

\section{Singular values}

Given a complete matrix $A$. The maximum singular value of this matrix is defined as

$$
\bar{\sigma}(A)=\max _{i} \sqrt{ } \lambda_{i}\left(A^{*} A\right)
$$


and the minimum singular value as

$$
\underline{\sigma}(A)=\min _{i} \sqrt{ } \lambda_{l}\left(A^{*} A\right)
$$

where $\lambda_{t}(\cdot)$ denotes an eigenvalue of the argument matrix.

Singular values have proved to be of great importance in the analysis and design of robust control systems (Doyle and Stein 1981, Lee et al. 1982, Lehtomaki et al. 1981 a, b, MacFarlane 1981). It can, for example, be shown (Lee et al. 1982) that in a stable feedback system $F$, stability will be maintained as perturbations $d F$ are added, provided that

$$
\underline{\sigma}[j \omega I-F]>\bar{\sigma}(d F)
$$

In general this expression has to be developed as function of the frequency $\omega$. We may, however, simplify the situation considerably if we assume that the feedback system $F$ has real eigenvalues only. With the design method for LQR mentioned above, this is almost always possible.

Assuming real eigenvalues in $F$, we get the condition

$$
\underline{g}(F) \leqslant \underline{\sigma}(j \omega I-F) \text { for all } \omega
$$

One way to see this is to look at the eigenvalue matrix $\Lambda=M^{-1} F M$. With $\Lambda$ we get

$$
\underline{\sigma}(j \omega I-\Lambda)=\min _{i} \sqrt{ }\left(\lambda_{i}^{2}+\omega^{2}\right)
$$

and thus

$$
\underline{\sigma}(\Lambda) \leqslant \underline{\sigma}(j \omega I-\Lambda)
$$

Since $F$ and $\Lambda$ are similar matrices, we conclude from (20) that (18) also must be valid.

Combining (17) and (18) yields the condition for stability

$$
\underline{\sigma}(F)>\bar{\sigma}(d F)
$$

This is a very useful condition as it permits the study of a large variety of perturbations without too much computational efforts. The drawback with this analysis, as with all analysis involving singular values, is the inherent conservatism of the method.

If we instead of considering absolute stability, consider $\alpha$-stability (all eigenvalues to the left of $-\alpha$ on the real axis), condition (21) is changed to

$$
\underline{\sigma}(F+\alpha I)>\bar{\sigma}(d F)
$$

This may also be written

$$
\underline{\sigma}(F)>\bar{\sigma}(d F)+\alpha
$$

or

$$
\bar{\sigma}(d F)<\underline{\sigma}(F)-\alpha
$$

Using (23) or (24) gives, however, a more conservative result than (22).

\section{Example 4}

Given the same system as in Example 2. We compute

$$
\underline{\sigma}(F)=\underline{g}(A+B G)=4 \cdot 53
$$


The eigenvalues of $F$ are -5 and -8 . Specifying $\alpha=4$, we would like to know what percentage variations in the elements of $A$ can be tolerated. We write

$$
d A=\left[\begin{array}{ll}
-2 \epsilon & 0 \\
-\epsilon & \epsilon
\end{array}\right]
$$

The sign of the variations is the same as in Example 2.

$$
\bar{\sigma}(d F)=\bar{\sigma}(d A)=2 \cdot 29 \epsilon
$$

With $\alpha=4$

Using (22) we get

$$
\underline{\sigma}(F+4 I)=0 \cdot 79
$$

$$
0 \cdot 79>2 \cdot 29 \epsilon
$$

or

$$
\epsilon<0 \cdot 345
$$

That is, a $34.5 \%$ change in the elements of $A$ can be tolerated. With

$$
d A=\left[\begin{array}{ll}
-0.69 & 0 \\
-0.345 & 0.345
\end{array}\right]
$$

the eigenvalues of the closed-loop system become $-4 \cdot 36$ and $-8 \cdot 99$. As expected, the result is somewhat conservative.

If we instead of condition (22) use condition (24), we get

$$
2 \cdot 29 \epsilon<(4 \cdot 53-4)=0.53
$$

or

$$
\epsilon<0 \cdot 23
$$

As expected, this result is still more conservative.

For the analysis of a system with state estimator we use eqns. (7) and (9) and condition (21)

$$
\underline{\sigma}(\hat{F})>\bar{\sigma}(d \hat{F})
$$

\section{Example 5}

Using the same system as in Example 3 we get

$$
\underline{\sigma}(\hat{F})=1 \cdot 27
$$

We note that this value is considerably smaller than for the same system without estimator $(\sigma(F)=4.53)$ indicating that smaller perturbations can be tolerated.

With the same $d A$ as in Example 4 we get

$$
\bar{\sigma}(d \hat{F})=\bar{\sigma}(d A)=2 \cdot 29 \epsilon
$$

With $\alpha=4$

$$
\underline{\sigma}(\hat{F}+4 I)=0 \cdot 23 \text { giving } \epsilon \leqslant 0 \cdot 1
$$

The allowed percentage perturbations are $10 \%$ as compared with $34.5 \%$ for the system without estimator. 


\section{The Block Gerschgorin theorem}

The block Gerschgorin theorem, which is a generalization of the Gerschgorin circle theorem, is useful in the study of decentralized control systems (Solheim 1981). It may, however, also be used in the study of perturbed systems as will be shown below.

Consider a system matrix $F$ with perturbations $\pm d F$. We establish an augmented system matrix

$$
\left[\begin{array}{c:c}
F+d F & 0 \\
\hdashline 0 & F-d F
\end{array}\right]
$$

We use the similarity transformation

$$
\left.\left.\left[\begin{array}{c:c}
I & I \\
\hdashline I & -I
\end{array}\right]\right] \begin{array}{c:c}
F+d F & 0 \\
\hdashline 0 & F-d F
\end{array}\right]\left[\begin{array}{c:c}
-I & -I \\
\hdashline-I & I
\end{array}\right]=\left[\begin{array}{c:c}
F & d F \\
\hdashline d F & F
\end{array}\right]=\tilde{F}
$$

The eigenvalues of $\tilde{F}$ consist of the eigenvalues of the original system $F$ with perturbations $+d F$ and those of $F$ with perturbations $-d F$. In order that $\tilde{F}$ be in a form that is suitable for applying the block Gerschgorin theorem, we have to modify it to make the block diagonal matrices normal. (A matrix is called normal if it commutes with its conjugate transpose.) The easiest way to do this is to use diagonalization:

$$
\left[\begin{array}{c:c}
M^{-1} & 0 \\
\hdashline 0 & M^{-1}
\end{array}\right]\left[\begin{array}{c:c}
F & d F \\
\hdashline d F & F
\end{array}\right]\left[\begin{array}{c:c}
M & 0 \\
\hdashline 0 & M
\end{array}\right]=\left[\begin{array}{c:c}
\Lambda & M^{-1} d F M \\
\hdashline M^{-1} d F M & \Lambda
\end{array}\right]=F^{*}
$$

where $\Lambda$ is the diagonal eigenvalue matrix of $F$ and $M$ is an eigenvector matrix of $F$. (Distinct real eigenvalues are assumed.)

The block Gerschgorin theorem states (Solheim 1981).

The inclusive regions for the eigenvalues of the matrix $F^{*}$ (and thus also of $\tilde{F}$ ) consist of circles with centres at the eigenvalues of $F$ and with radius equal to the Euclidean norm $\left\|M^{-1} d F M\right\|_{2}=\bar{\sigma}\left(M^{-1} d F M\right)$.

The condition for stability becomes therefore, remembering that all eigenvalues of $F$ are real:

$$
\bar{\sigma}\left(M^{-1} d F M\right)<|\lambda(F)|_{\text {min }}
$$

This condition may now be compared with condition (21).

\section{Example 6}

Given the same system and perturbations as in Example 4. The eigenvalues of the closed-loop system $F$ are -5 and -8 . The radius of the circles defining the inclusion regions for the eigenvalues of the perturbed system

$$
r=\bar{\sigma}\left(M^{-1} d A M\right)=5 \cdot 12 \epsilon
$$

With $\epsilon=0 \cdot 1(=10 \%)$ we get the inclusion regions shown in Fig. 1. The eigenvalues of $(F+d F)$ and $(F-d F)$ are also shown in the figure. As expected, we get a somewhat conservative result. 


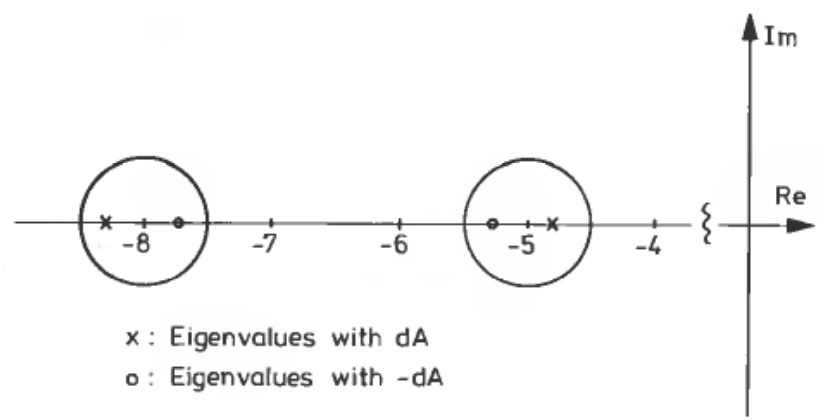

Figure 1. Inclusion regions for Example 6.

\section{Example 7}

Given the same process as in Example 1. Consider the following perturbations

$$
d F=d A=\left[\begin{array}{rrr}
0 & -\epsilon & 0 \\
0 & 0 & 0 \\
0 & 0 & -\epsilon
\end{array}\right]
$$

We use the same controllers as in Example 1.

(1) LQR with prescribed eigenvalues (Solheim 1972).

(2) A controller minimizing some of the eigenvalue sensitivies (Gourischankar and Ramar 1976).

(3) A controller making some eigenvalues invariant to parameter perturbations (Shah et al. 1977).

For these three controllers we compute the radius of the inclusion regions with the perturbations stated above.

Controller 1: $r=4 \cdot 71 \epsilon$

Controller 2: $r=7 \cdot 88 \epsilon$

Controller 3: $r=8 \cdot 96 \epsilon$

The radius $r$ will to some extent indicate how sensitive the eigenvalues are to the assumed parameter variations. We note that controller 1 is better in this respect than the others.

Figure 2 shows the inclusion regions for the different controllers with $\epsilon=0 \cdot 1$. The eigenvalues for the systems $(F+d F)$ and $(F-d F)$ are also shown in the figure.

\section{Concluding remarks}

We have demonstrated the use of three different methods for analysing the robustness of optimal control systems. The analysis is somewhat simplified by assuming only real eigenvalues of the closed-loop system.

Using eigenvalue sensitivity coefficients we have constructed a set of 'worst case' perturbations, and used these to study the stability of the system. The use of singular 

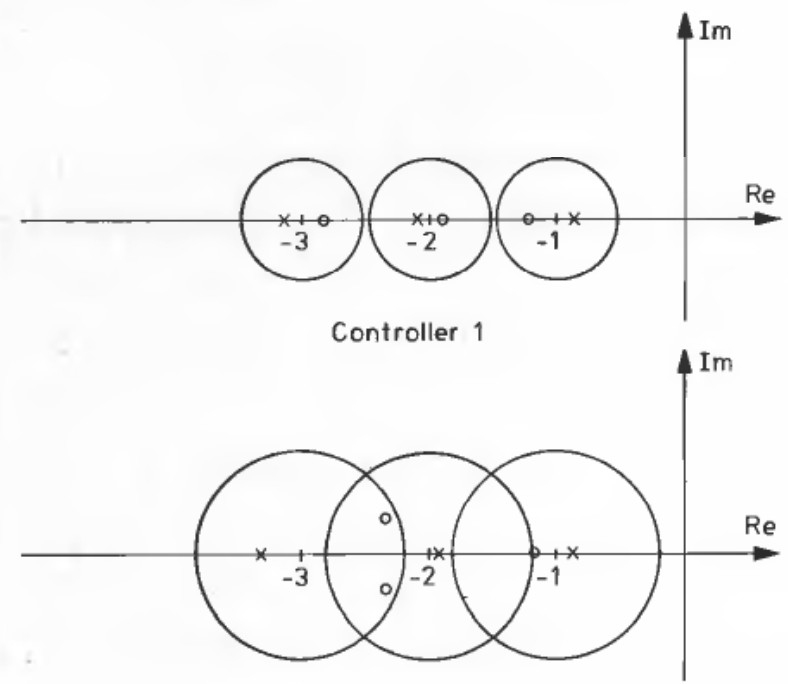

Controller 2

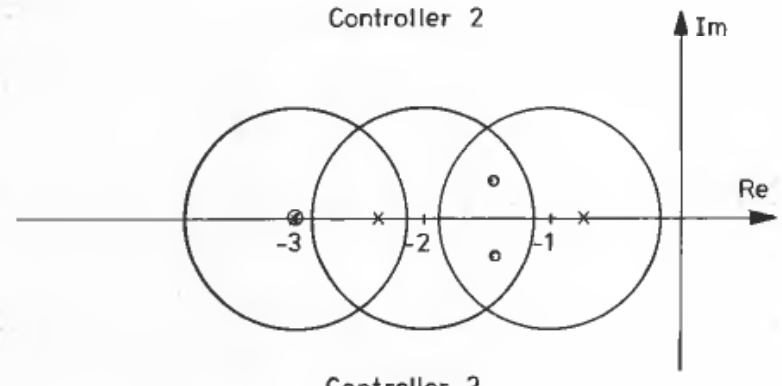

Controller 3

$x$ : Eigenvalues with dA

$\circ$ : Eigenvalues with - dA

Figure 2. Inclusion regions for Example 7.

values permits greater freedom in the structure of the perturbations but, on the other hand, also gives a somewhat conservative result. The block Gerschgorin theorem enables us to construct inclusion regions for the eigenvalues in the presence of parameter perturbations. All the methods discussed are also useful when comparing the robustness properties of different types of controllers.

\section{REFERENCES}

ANDERSEN, B. D. O., and MOORE, J. B. (1971). Linear optimal cantrol (Prentice-Hall).

DOYLE, J. C., and STEIN, G. (1981). Multivariable feedback design: Concepts for a classical/ modern synthesis. IEEE Trans. autom. Control, 26, 4-16.

FADDEEv, D. K., and FADDEeva, V. N. (1963). Computational methods of linear algebra, (W. H. Freeman \& Co.).

GRAUPE, D. (1972). Derivation of weighting matrices towards satisfying eigenvalue requirements, Int. J. Control, 16, 881-888.

GourischanKar, V., and Ramar, K. (1976). Pole assignment with minimum eigenvalue sensitivity to plant parameter variations. Int. J. Control, 23, 493-504. 
Heger, F., and Frank, P. M. (1982). Computer-aided pole placement for the design of robust control systems, IFAC Symposium on Computer aided design of multi-variable technological systems, Purdue Univ., Lafayette, Ind., Sept. 15-17, 1982.

Howze, J. W., and CARIN, III, R. K. (1979). Regulator design with nodal insensitivity, IEEE Trans. autom. Control, 24, 466-469.

Lehtomakı, N. A., SANDell, N. R., and AthanS, M. (1981 a). Robustness results in linearquadratic Gaussian based multivariable design. IEEE Trans. autom. Control, 26, 75-93.

LEHTOMAKı, N. A., et al. (1981 b). Robustness tests utilizing the structure of modelling errors. 20th CDC, San Diego, Dec. 16-18.

Lee, W. H., Gully, S. W., Eterno, J.S., and SANDEll, N. R. (1982). Structural information in robustness analysis. American Control Conference (ACC), Arlington, Va, June 14-16, 1982.

MacFarlane, A. G. J. (1981). Characteristic and principal gains and phases and their use as multivariable control design tools. AGARD Lecture series No. 117, Paper no. 2, 1-34.

Sambandan, A., and Chandrasekharan, P. C. (1981). Design of output feedback controller with eigenvalue and eigenvector intensitivity. Int. J. Control, 33, 935-943.

Shah, S. L., Fisher, D. G., and SeborG, D. E. (1955). Eigenvalue invariance to system parameter variations by eigenvector assignment. Int. J. Control, 26, 871-881.

Solheim, O. A. (1981). On the use of a block analogue of the Gerschgorin circle theorem in the design of decentralized control of a class of large scale systems, 2nd IFAC symposium on large scale systems, Toulouse, June 24-26, 1980. Modeling, Identification and Control, 2, 107-118.

Solmeim, O. A. (1980). On the use of low-order Riccati equations in the design of a class of feedback controllers and state estimators, Modeling, Identification and Control, 1, 231-245.

Solheim, O. A. (1972). Design of optimal control systems with prescribed eigenvalues, Int. J. Control, 15, 142-160.

Solheim, O. A., and SAlid, S. (1971). Eigenvalue sensitivity in optimal feedback control systems, 2nd IFAC Symposium on Multivariable technical control systems, Düsseldorf, Oct. 11-13, 1971.

TAYLOR, J. S., and TUTEUR, F. B. (1966). The use of a quadratic perturbance index to design multivariable control systems, IEEE Trans. autom. Control, 11, 84-92. 